\title{
Review of: "Magnetic memory driven by topological insulators"
}

\author{
Wen Jeng Hsueh ${ }^{1}$ \\ 1 National Taiwan University
}

Potential competing interests: The author(s) declared that no potential competing interests exist.

This work experimentally investigated the SOT-MRAM by using the topological insulator $\left(\mathrm{BiSb}_{2} \mathrm{Te}_{3}\right)$, in which the charge-spin conversion efficiency is largely enhanced. Two charge-spin conversion efficiencies, 1.59 and 1.02, are achieved by using the SOT-induced shift of the magnetic field and the ST-FMR, respectively. The TMR ratio of $102 \%$ and the low switching current density of $0.12 \mathrm{MA} \mathrm{cm}^{-2}$ at room temperature have been proposed. The manuscript will be probably suitable for publication after revising the follow questions:

(1) The results of charge-spin conversion efficiency larger than 1 is at first sight very intriguing, but such a TI-driven SOT-MRAM is hard to be made in industrial manufacture, as mentioned on the page 4 . The ultrahigh charge-spin conversion efficiency is achieved by growing the $\mathrm{TI}$ on the substrate by the MBE method. If the $\mathrm{Tl}$ is constructed by the magnetron sputtering method, the switching current density isn't low anymore, that is to say, the charge-spin conversion efficiency is unable to exceed 1. Did you measure the charge-spin conversion efficiency of the sputtered TI-driven MTJ? Is the high conductivity the only advantage of the sputtered TI-driven MTJ?

(2) This work uses two different ways, the SOT-induced shift of the magnetic field and the ST-FMR, to get the charge-spin conversion efficiency. However, the difference of the two values of the charge-spin conversion efficiency is huge. Which value is more reliable? What is the reason for having such a big difference between the two kinds of measurements?

(3) In general, the coercive field in fixed layer is larger than that in free layer. However, the statement on the page 2 says that the coercive field of T-CoFeB and B-CoFeB are 10Oe and 20Oe, respectively. What is the reason for the free layer with lager coercive field? Is it a typographical error?

(4) Size of the MTJ is scaling down from 4um*8um to $100 \mathrm{~nm} * 200 \mathrm{~nm}$, which is consistent with the grain size of the TI surface. However, the aspect ratio (AR) isn't unchanging during the process of scaling down in figure 2(e). The AR is associated with shape anisotropy which changes the $J_{C}$ of the free layer in in-plane type. Did you consider the influence of different shapes of the MTJ? 
(5) The definition of switching ratio is ambiguous on the page 3. From the article, readers are unable to understand the physical meaning of $\triangle T M R / T M R$ and having no idea how to calculate $\triangle T M R$. Where does the $\triangle T M R$ come from? As shown in figure 2(e), what is the reason for having the relationship between $\triangle T M R$ and area of free layer?

(6) The name of the material within the black area between Ru and $\mathrm{BiSb}_{2} \mathrm{Te}_{3}$ should be marked clearly in the schematic of STEM, which can help the readers recognize the clear interface between TI and MTJ mentioned in the article. 